\title{
INCLUSÃO DIGITAL EM ESCOLAS PÚBLICAS ATRAVÉS DE TECNOLOGIAS INOVADORAS DE BAIXO CUSTO NO ENSINO DE DISCIPLINAS STEM
}

\author{
Isabela Nardi da Silva - isabela.nardi@hotmail.com - PPGTIC/UFSC \\ Karmel Cristina Nardi da Silva - karmelnardi@hotmail.com - PPGTIC/UFSC \\ Karen Schmidt Lotthammer - lotthammer_karen@hotmail.com - PPGTIC/UFSC \\ Juarez Bento da Silva - juarez.b.silva@ieee.org - UFSC \\ Simone Meister Sommer Bilessimo - simone.bilessimo@ufsc.br - UFSC
}

\begin{abstract}
Resumo. O projeto "Promovendo inclusão digital em escolas públicas de educação básica através da integração de tecnologias inovadoras de baixo custo no ensino de ciências naturais e exatas" tem sido realizado pelo Laboratório de Experimentação Remota (RExLab), da Universidade Federal de Santa Catarina, desde 2008. Este projeto já capacitou 363 docentes de 6 escolas, uma vez que se trata de uma iniciativa de integração de tecnologia na educação básica da rede pública de ensino brasileira. O presente estudo foca nos benefícios do projeto em relação aos docentes de educação básica envolvidos em seu âmbito. Os resultados positivos adquiridos pelo projeto ao longo dos anos demonstram que a integração de tecnologias na educação básica por parte dos docentes na rede pública é uma prática a ser incentivada.
\end{abstract}

Palavras-chaves: capacitação docente; tecnologias da informação e comunicação; ambiente virtual de aprendizagem.

\section{DIGITAL INCLUSION IN PUBLIC SCHOOLS THROUGH INTEGRATION OF INNOVATIVE TECHNOLOGIES OF LOW COST IN TEACHING OF STEM SUBJECTS}

Abstract. The project "Promoting digital inclusion in public schools of basic education through the integration of innovative low-cost technologies in the teaching of natural and exact sciences" has been carried out by the Remote Experimentation Laboratory (RExLab), Federal University of Santa Catarina, since 2008, having trained 363 teachers of 6 schools. The article focuses on the benefits of the project in relation to the basic education teachers involved in its scope. The project is an initiative of technology integration in the basic education of the Brazilian public school system. The positive results acquired by the project over the years demonstrate that the integration of technologies in basic education by teachers in the public network is a practice to be encouraged.

Keywords: teacher training, information and communication technologies; virtual learning environment.

\section{Introdução}

As Novas Tecnologias da Informação e Comunicação são capazes de trazer benefícios para diversas áreas, e dentre estas se encontra a educação; através da integração de tecnologia na educação, o conceito de sala de aula torna-se maior, uma vez que o acesso à Internet favorece o uso de mídias como vídeos online, imagens, podcasts, games, entre outros (HENTON, 2016). Apesar de existir uma resistência inicial em relação ao uso de tecnologia em sala de aula da parte de muitos professores por conta de estes possuírem baixo conhecimento em relação às novas tecnologias, o 
uso de tecnologia tem muito a favorecer no trabalho dos docentes, podendo otimizar muitas de suas funções e torná-las mais fáceis, como correção de trabalhos escolares, por exemplo (PRESBY, 2017).

Neste âmbito, foi iniciado em 2008 o projeto "Promovendo inclusão digital em escolas públicas de educação básica através da integração de tecnologias inovadoras de baixo custo no ensino de ciências naturais e exatas". Este projeto foi iniciado em 2008 pelo Grupo Temático em Experimentação Remota Móvel (GT-MRE), do Laboratório de Experimentação Remota (RExLab), pertencente à Universidade Federal de Santa Catarina, no Brasil, e contando principalmente com a participação de docentes e estudantes do Programa de Pós-Graduação em Tecnologias da Informação e Comunicação, bacharelado em Tecnologias da Informação e Comunicação e bacharelado em Engenharia de Computação.

O projeto foi criado com o objetivo de oferecer uma iniciativa para integração de tecnologias digitais na educação básica da rede pública brasileira, com vista na carência enfrentada por grande parte das escolas públicas brasileiras não possuírem laboratórios de ciências ou de informática. Para realizar este objetivo, o projeto foi estruturado em dois eixos. Um eixo formativo, que visa a capacitação docente em relação a tecnologias digitais, para que os professores aprendam como utilizar tecnologias bem como saber aplicá-las em aula, e, após um eixo integrativo, durante o qual professores realizam aplicações de tecnologias digitais em sala de aula, com seus estudantes.

Com o passar dos anos, o projeto tem apresentado resultados muito positivos, sendo comprovados mediante da satisfação de docentes e estudantes envolvidos em suas ações e por conta da forma como estes expuseram suas opiniões em questionários aplicados durante o projeto.

Desta forma, o presente trabalho possui como objetivo apresentar o projeto com foco nos benefícios que este traz aos docentes que são participantes dele.

\section{Materiais e Métodos}

\subsection{Procedimentos Metodológicos}

A presente pesquisa foi realizada a partir da utilização da abordagem qualitativa, que de acordo com Gil (2007), é percebida através de uma compreensão de um grupo social para obter o entendimento da totalidade do fenômeno. Segundo Minayo (2001), este tipo de pesquisa trabalha com uma versão mais profunda dos fenômenos, considerando suas variáveis, ideal para ambientes educacionais. Prodanov e Freitas (2013) defendem que a pesquisa qualitativa permite considerar a relação dinâmica entre o mundo real e o sujeito, percebendo seu vínculo inseparável e percebendo que o mesmo não poderia ser traduzido em números.

Quanto a natureza, a presente pesquisa pode ser definida como pesquisa aplicada, pois se direciona para a solução de problemas de maneira específica. Prodanov e Freitas (2013), consideram que a pesquisa aplicada envolve verdades e interesses locais, solucionando os mesmos de maneira prática e explorando diferentes fatores pertencentes ao assunto em questão.

Com relação aos objetivos, este estudo se enquadra na pesquisa exploratória, que de acordo com Gil (2007) é ideal para esclarecer melhor determinado problema e a construção de suas hipóteses, ou seja, objetiva ampliar a familiaridade com a problemática em questão. Para Prodanov e Freitas (2013), este tipo de pesquisa facilita a delimitação do tema, por explorar de forma mais ampla suas hipóteses através de um planejamento flexível. Muitas vezes as pesquisas exploratórias assumem formas de pesquisas bibliográficas ou estudos de caso. 
Considerando os procedimentos, a pesquisa se trata de um estudo de caso, por estudar especificamente um grupo. De acordo com Fonseca (2002), o estudo de caso surge para estabelecer uma compreensão acerca de uma situação diferenciada em diversos aspectos, buscando encontrar suas características particulares. Este tipo de estudo apresenta uma perspectiva geral da temática a partir da visão do investigador.

\subsection{Desenvolvimento e aplicação do curso semipresencial}

O curso semipresencial é denominado "Capacitação Docente para Integração do uso das TIC na Educação Básica", sendo disponibilizado na plataforma InTecEdu (http://intecedu.ufsc.br/), desenvolvida pelo RExLab. Esta plataforma é um ambiente virtual de aprendizagem baseado no sistema MOODLE, e através dela são disponibilizados diversos cursos, tanto capacitação docente quanto cursos criados por professores já certificados por este - ação que se encontra no eixo integrativo da capacitação docente, momento em que professores aplicam de fato as tecnologias educacionais aprendidas no momento de capacitação.

No total, o curso de capacitação docente possui duração de $120 \mathrm{~h}$, sendo que $90 \mathrm{~h}$ devem ser realizadas através de atividades executadas no ambiente virtual de aprendizagem e $30 \mathrm{~h}$ destas horas são executadas através de atividades presenciais, como aulas práticas e palestras lecionadas pelo bolsista de extensão responsável pela escola e outros integrantes da equipe do RExLab, inclusive docentes parceiras do projeto já certificadas pelo curso. Além de completar $120 \mathrm{~h}$, para certificação é necessário que o docente cumpra pelo menos $75 \%$ das atividades propostas; após a realização do curso, o docente recebe um certificado de curso de extensão de $120 \mathrm{~h}$. Em relação às unidades do curso, este possui 6 unidades, a serem apresentadas nos parágrafos a seguir.

A primeira etapa do curso foi de apresentação, funcionando como uma espécie de introdução, a fim de explicar os objetivos deste, bem como detalhes como sua modalidade, duração do curso e detalhamento desta. Além disso, esta etapa contou com a aplicação de dois questionários, no formato de enquete: Pesquisa TPACK e Perfil Docente. Através da pesquisa TPACK, professores informaram sua familiaridade com tecnologias e seu nível de dificuldade em relação a seu manuseio. Esta pesquisa possuía 35 questões. Em relação ao questionário de Perfil docente, este era referente ao perfil individual do professor, apresentando questões relacionadas ao seu gênero, faixa-etária, escolaridade, entre outras.

Após a apresentação do curso, os professores acessam a Unidade 1, onde realizam a avaliação inicial do curso, feita de modo a comparar a diferença do conhecimento do professor antes de começar o curso e depois de terminá-lo - na última unidade, existe um questionário idêntico, a ser respondido novamente pelos docentes. Além disso, existe um material para leitura.

A Unidade 2 é denominada "A plataforma educacional MOODLE", e é o momento em que professores aprendem como editar um curso no MOODLE. A partir desta unidade, todos os módulos são divididos em três etapas: a avaliação inicial da unidade em questão, o conteúdo didático para leitura e atividades para submissão e a avaliação final da unidade. Assim como as avaliações iniciais e finais do curso em geral, cada unidade as possui individualmente, a fim de verificar o quanto o professor pôde aprender com os exercícios e informações apresentadas na unidade.

A unidade seguinte é referente ao "Planejamento e publicação de recursos do MOODLE e da WEB 2.0", momento em que o docente aprende a incorporar em sua aula virtual os diversos recursos que o MOODLE oferece e também da WEB 2.0 como material alternativo. Este módulo possui o mesmo formato da Unidade 2, sendo iniciado 
com a avaliação inicial, seguido das atividades e material para leitura e a avaliação final da unidade.

Com estrutura semelhante às duas unidades anteriores, a Unidade 4 possui como pressuposto apresentar o "Planejamento e criação de atividades educacionais no MOODLE", a fim de que os participantes tenham conhecimentos e habilidades para gerar atividades educacionais utilizando as ferramentas que o MOODLE oferece.

Seguindo a estrutura das unidades anteriores, a Unidade 5 é denominada "Planejamento e criação de espaços de comunicação no MOODLE", objetivando que os docentes tivessem conhecimento para gerar espaços de aprendizagem, comunicação e socialização entre os demais participantes e com seus alunos futuramente.

Por fim, era apresentada a Unidade 6, denominada "Criação e configuração de Laboratório de Avaliação", criada a fim de que os participantes tivessem conhecimentos para gerar laboratórios de avaliação, tornando possível avaliação por pares. Por ser a última unidade, esta também contou com a avaliação final do curso, momento em que os conhecimentos prévios dos participantes são comparados com os conhecimentos póscurso.

\section{Referencial Bibliográfico}

\subsection{Deficiências de infraestrutura em escolas públicas de ensino básico}

De acordo com Silva e Navarro (2012) aprender é ampliar o conhecimento já existente por meio de desafios e confronto de saberes e para que isso aconteça, é necessário criar um meio para tal. Neste sentido, para que o aluno aprenda é fundamental que ele pratique as informações recebidas em sala de aula, a fim de consolidar o seu aprendizado.

Entretanto, para que os alunos possam realizar as suas práticas, é necessária infraestrutura física ou algum meio seja ele físico ou digital que a possibilite. Dados do Censo Escolar/INEP do ano de 2016 indicam que somente $42 \%$ das escolas brasileiras possuem laboratórios de informática, contando com cerca de 8 computadores para uso dos alunos, por escola, e $11 \%$ das instituições possuem laboratórios de ciências para práticas dos alunos. De acordo com a mesma pesquisa, apenas 56\% das escolas possuem Internet do tipo banda larga, disponível para uso de professores, alunos e direção.

Tendo em vista as deficiências de infraestrutura das instituições de ensino básico no Brasil e a disseminação de uso de dispositivos móveis na sociedade, observa-se no uso das TIC uma forma de suprir a infraestrutura física precária ou inexistente, e ainda, fazer com que o contexto conectado vivido pelo aluno se aproxime do contexto de sala de aula.

Para a integração das TIC no processo de ensino e aprendizagem, os docentes têm um papel fundamental, pois mais importante que o aluno aprender a fazer uso de dado programa, é encontrar formas de utilizá-lo didaticamente no processo de ensino e aprendizagem (RODRIGUES, 2016). Entretanto, ao buscar a utilização das TIC em sala de aula, os professores encontram algumas barreiras: infraestrutura precária, falta de suporte técnico e de instruções para o uso da tecnologia.

Segundo pesquisa realizada pelo Centro Regional de Estudos para o Desenvolvimento da Sociedade da Informação (CETIC.BR, 2017) do ano de 2016, $69 \%$ dos docentes entrevistados alegaram não utilizar o laboratório de informática da escola, fator em parte ocasionado devido à falta de computadores disponíveis para uso. De acordo com a mesmo estudo, os docentes apontaram algumas barreiras que segundo eles, dificultam muito o uso das TIC nas escolas: $69 \%$ elencaram o número 
insuficiente de computadores conectados à Internet; $61 \%$ indicaram a ausência de suporte técnico e manutenção dos equipamentos e $49 \%$ citaram a falta de um curso específico para o uso das TIC em sala de aula como fator de entrave.

À vista disso, a falta de infraestrutura de laboratórios de informática torna-se uma grande barreira para integração das TIC no ensino e para a prática dos discentes. Entretanto, o uso de recursos tecnológicos como dispositivos móveis, por exemplo, torna-se uma forma de suprir essa falta (ABULGAMI, 2016), possibilitando aos alunos a prática por meio de seus próprios dispositivos fazendo uso de aplicativos, simulações ou experimentos remotos que podem ser acessados a partir de qualquer hora e lugar.

\subsection{Formação continuada de docentes da educação básica}

A integração das TIC no cotidiano das pessoas reflete-se também na educação, promovendo a adoção de novas ferramentas no processo educacional (NICOLETE et. al., 2015). Entretanto, diversos fatores dificultam a inclusão das TIC no processo de ensino e aprendizagem, dentre elas: políticas públicas descontinuadas, inexistentes ou equivocadas, visão ultrapassada quanto ao currículo educacional, falta de instruções dos docentes e sua grande carga horária exercida, além da resistência à mudança (MALTEMPI; MENDES, 2016).

Tendo em vista a grande utilização de dispositivos móveis pelos alunos e o constante acesso a inúmeras informações por meio deste, o docente passa a possuir um papel de extrema importância no uso educacional da tecnologia, orientando aos seus alunos para fazer uso dela em prol do seu aprendizado. Assim, o processo de ensino e aprendizagem em que o docente é o detentor do conhecimento e os alunos apenas expectadores, passa a deixar de existir (OLIVEIRA; FRANCO JUNIOR, 2016).

Dar instruções aos docentes de como fazer uso pedagógico das TIC em suas práticas torna-se fundamental para integração às TIC na educação. De acordo com a Organização das Nações Unidas para a Educação, a Ciência e a Cultura (UNESCO, 2008) os docentes deveriam possuir algumas competências na área de TIC:

- Fazer uso dos recursos das TIC para melhorar sua produtividade, para aquisição de conhecimento pedagógico e da matéria;

- Utilizar os laboratórios de informática nas atividades de ensino em processo;

- Usar a rede para apoiar a colaboração do aluno dentro e fora de sala de aula.

Entretanto, segundo pesquisa realizada pelo Centro Regional de Estudos para o Desenvolvimento da Sociedade da Informação (Cetic.br) no ano de 2016, $70 \%$ dos docentes citaram não ter realizado um curso de formação continuada, fazendo com que as competências indicadas pela Unesco (2009) se tornem distantes da realidade brasileira.

À vista disso, os dados acima indicam a necessidade de formação continuada de docentes para que estes, possuam instruções de como integrar as TIC em suas práticas e que possam também, orientar adequadamente seus alunos para o uso pedagógico desta.

\section{Histórico do projeto "Promovendo inclusão digital em escolas públicas de educação básica através da integração de tecnologias inovadoras de baixo custo no ensino de ciências naturais e exatas"}

O projeto está relacionado a uma série de pequenos projetos em desenvolvimento e em constante evolução ao longo dos anos. Em 2005, o RExLab obteve, juntamente com outras instituições de ensino superior parceiras a aprovação do projeto RExNet - Yippee (Remote Experimentation Network - Yielding na inter-university peer-to-peer eservice), junto ao Programa Alfa da Comunidade Europeia. O projeto contou com a participação de 12 instituições de ensino superior de sete países (6 europeias de 4 países 
e 6 latino-americanas de 3 países) e foi escolhido como "case" dentro da referida convocatória.

O sucesso e o caráter inovador do projeto RExNet motivou o proponente desta a apresentar uma proposta junto ao Conselho Nacional de Desenvolvimento Científico e Tecnológico (CNPq), com o objetivo de obter recursos, pois, embora o projeto RExNet tivesse seu período formal encerrado junto ao Programa Alfa II, as parcerias se mantiveram e seria possível capitalizar no Brasil parte da experiência e expertise construída no projeto. Assim, em 2007 foi submetida ao CNPq uma proposta relacionada a utilização da experimentação remota como suporte à ambientes colaborativos de aprendizagem. Porém, o resultado final em relação à proposta foi "Indeferida" e não foi obtido feedback algum.

Em contrapartida, em 2007 foi enviada a proposta de projeto "Utilização da experimentação remota como suporte a ambiente de ensino-aprendizagem na rede pública de ensino" a uma convocatória do Fundo Regional para Inovação Digital na América Latina e Caribe (FRIDA). Neste caso, o projeto foi aprovado e obteve recursos na ordem de US\$12.400. O sucesso no desenvolvimento no projeto foi formalizado em convite para apresentar o projeto no "I Encuentro FRIDA: Investigaciones em Tecnologías de la Informacion y Comunicacion y politicas publicas en America Latina y el Caribe", em Montevidéo, na mesa redonda "Experiencias de investigaciones exitosas financiadas for FRIDA".

Em 2011, o projeto "Utilização da experimentação remota como suporte a ambiente de ensino-aprendizagem na rede pública de ensino" foi selecionado como um dos quatro projetos mais inovadores na educação brasileira. A seleção foi realizada pelo Instituto para o Desenvolvimento e a Inovação Educativa (IDIE), da Organização dos Estados Ibero-Americanos para a Educação, a Ciência e a Cultura (OEI) e patrocinado pela Fundação Telefônica. O estudo foi realizado em âmbito nacional, e foram identificados 64 projetos, inicialmente considerados relevantes sobre a inovação em educação com o uso de TIC, onde 26 foram caracterizados como realmente inovadores, e, destes, quatro projetos foram considerados de vanguarda.

No mesmo ano, este projeto foi um dos escolhidos para representar o Brasil no "VI Encuentro Internacional EducaRed 2011”. Em fevereiro de 2012, foi tema em debate promovido no evento brasileiro Campus Party em mesa de debates intitulada "Mobilidade digital e educação: a escola para além de seus muros", que teve como objetivo trazer a reflexão sobre uma nova forma de pensar a educação - a partir da apresentação de diferentes práticas de uso de dispositivos móveis, como smartphones e laptops para fins educacionais.

Em abril de 2014, foi aprovado o projeto "Utilização de Experimentação Remota em Dispositivos Móveis para a Educação Básica na rede pública de ensino", junto ao FRIDA, na convocatória "Escalamientos Frida 2014", onde obteve aprovação de recursos na ordem de US\$13, 951,00 que foram aplicados em material permanente no projeto, com duração de um ano.

Atualmente, o Laboratório de Experimentação Remota possui como principal projeto relacionado a capacitação docente em escolas da rede pública de ensino o programa "Promovendo a inclusão digital em escolas de Educação Básica da rede pública a partir da integração de tecnologias inovadoras de baixo custo no ensino de Ciências Naturais e Exatas". Este projeto é realizado em parceria com a Universidade Federal de Santa Catarina, o Ministério da Educação Brasileiro, a Rede Nacional de Ensino e Pesquisa (RNP), entre outros.

\section{Resultados e Discussão}


O curso de "Capacitação Docente para Integração do uso das TIC na Educação Básica" tem sido aplicado em seis escolas públicas localizadas no extremo sul de Santa Catarina. No município de Araranguá/SC, são atendidas quatro escolas: E.E.B. Castro Alves, E.E. Prof ${ }^{a}$ Maria Garcia Pessi, E.M. Otávio Manoel Anastácio e E.E.B. Araranguá. No município de Balneário Arroio do Silva/SC são atendidas duas escolas: E.E.B. Apolônio Ireno Cardoso e E.M.E.F. Jardim Atlântico.

Este curso é de formação contínua, sendo aplicado semestralmente nas escolas básicas parceiras; até a data de 01/10/2017, foram capacitados 363 docentes da rede pública. Levando em consideração que o Programa cujas ações concedem a capacitação docente é contínuo e o número de professores capacitado certamente será ampliado ao passar do tempo.

Em relação à maneira como a capacitação é realizada e como o professor aplica em sua rotina escolar os conhecimentos adquiridos, a experiência da utilização do ambiente virtual de aprendizagem como complementar no ensino é iniciada a partir do momento em que o professor solicita ao colaborador disponibilizado para a escola pelo Laboratório de Experimentação Remota (RExLab), instruções para a utilização da plataforma InTecEdu (http://intecedu.ufsc.br/).

A partir deste momento, o colaborador do RExLab presente na instituição apresenta ao professor o curso no MOODLE de Capacitação Docente para Integração do uso das TIC na Educação Básica, com duração de 120 horas, que é desenvolvido pelo RExLab e oferecido na própria escola, demonstrando os caminhos necessários para $o$ desenvolvimento de um trabalho satisfatório e possibilitando o aprendizado de ferramentas de trabalho úteis, como a plataforma MOODLE.

Utilizar a plataforma livre MOODLE permite que sites dinâmicos sejam desenvolvidos pelos docentes, possibilitando a gestão da avaliação dos alunos bem como o compartilhamento de materiais didáticos (ROCHADEL et. al, 2016). Desde o princípio da aplicação do curso de Capacitação Docente para Integração do uso das TIC na Educação Básica, o professor recebe suporte e assistência para suprir eventuais dúvidas, com o intuito de que possa estabelecer uma assimilação satisfatória do conteúdo, assegurando-se de que estará preparado para utilizar o recurso como uma alternativa para o complemento do ensino. Uma das importantes características da aplicação do curso em questão são as atividades práticas, que são construídas buscando familiarizar o professor com o conteúdo com o qual trabalhará.

Após o embasamento adquirido através da capacitação, o professor será capaz de gerar conteúdos para seus cursos, inserindo no MOODLE todos os itens necessários para complementar o conteúdo repassado em sala de aula. Para que o acesso possa ser efetuado o colaborador RExLab disponível na escola transportará tablets disponíveis no laboratório para a utilização em turma, visto que as instituições geralmente não possuem infraestrutura para que a aplicação possa ocorrer de maneira apropriada.

Os alunos utilizam os tablets para acessarem as sequências didáticas construídas pelo professor, após o mesmo discorrer sobre o conteúdo. Ao acessar o InTecEdu através dos tablets, os alunos descobrem vídeos, jogos e atividades correspondentes ao assunto exposto em sala de aula, sentindo-se extremamente motivados pela oportunidade de participarem de uma aula com uma perspectiva mais dinâmica que adentra o universo tecnológico ao qual estão inseridos na maior parte do tempo.

Os professores, que geralmente apresentam-se um pouco resistente no início da utilização, por considerar que integrar a tecnologia poderia ser um trabalho extra em sua rotina tão desgastante, por fim acabam considerando que ocorreu o contrário, devido ao fato da integração de tecnologia ter auxiliado no aproveitamento do seu tempo devido às ferramentas úteis do sistema, dentre elas alguns serviços automáticos como: correção de 
questionários na hora e conteúdo replicado para outras turmas que estejam estudando os mesmos assuntos.

\section{Conclusões}

O presente trabalho possui como objetivo apresentar os benefícios na performance dos docentes participantes do projeto "Promovendo inclusão digital em escolas públicas de educação básica através da integração de tecnologias inovadoras de baixo custo no ensino de ciências naturais e exatas", desenvolvido pelo RExLab há 9 anos. Estes docentes atuam na rede pública de ensino e estavam acostumados com a falta de recursos e infraestrutura com a qual instituições públicas de ensino brasileiras sofrem.

Por outro lado, existe uma infinidade de recursos tecnológicos de baixo custo e alta aplicabilidade em sala de aula, sendo alternativas com potencial para suprir eventuais déficits na educação básica pública. $\mathrm{O}$ grande problema é que muitos docentes não têm noção deste tipo de ferramenta pois possuem baixo conhecimento em relação a tecnologias digitais de forma geral. Desta forma, uma das ações do projeto desenvolvido pelo RExLab é de capacitar docentes participantes em relação a informática básica e ferramentas educacionais para uso em sala de aula.

Apesar da relutância inicial de muitos docentes participantes no início da capacitação, por conta de que estes acreditavam que aplicar tecnologia em sala de aula apenas lhe dariam mais trabalho para fazer, estes logo perceberam que estavam enganados; afinal, mediante a capacitação perceberam que na verdade o uso de tecnologia lhes otimiza o tempo de trabalho, uma vez que atividades podem ser corrigidas automaticamente e materiais podem ser facilmente replicados para diversas turmas, por exemplo.

A motivação dos docentes tornou-se muito maior em relação ao uso de tecnologia em sala de aula, e tal motivação inclusive atingiu muitos de seus colegas, que se tornaram também interessados em iniciar o curso de capacitação docente. Isto demonstra que a capacitação desenvolve uma espécie de evento em corrente, em que a partir do momento em que o primeiro docente realiza a capacitação e apresenta a seus colegas os benefícios que obteve a partir desta, seus colegas se sentem motivados e passam a sentir interesse em aprender como integrar tecnologias em sala de aula.

Porém, a participação da escola também é importante neste processo. O diretor ou coordenador responsável pela instituição deve se manter parceiro ao projeto e incentiválo, de forma a realizar reuniões com os docentes da escola para acompanhar e discutir o uso de tecnologias e também com os coordenadores do projeto, a fim de informá-los acerca dos avanços e problemas encontrados durante as aplicações. Afinal, o projeto deve ocorrer de forma conjunta e colaborativa entre coordenadores do projeto, estagiários envolvidos, diretores de escola, equipe pedagógica, docentes participantes e os estudantes destes docentes. Desta forma, todos sempre estarão atualizados de acordo com os avanços e compartilharão soluções para eventuais problemas, e o trabalho será executado de forma natural e evolutiva, o que propiciará a elaboração de futuras pesquisas relacionadas a resultados do projeto.

\section{Agradecimentos}

O presente estudo foi financiado pelo Programa de Extensão Universitária (PROEX) da Universidade Federal de Santa Catarina e pelo Ministério da Educação (MEC), a Fundação de Apoio à Pesquisa e Inovação do Estado de Santa Catarina (FAPESC), a Coordenação de Aperfeiçoamento de Pessoal de Nível Superior (CAPES) e do Programa Institucional de Bolsas de Iniciação Científica do Conselho Nacional de Desenvolvimento Científico e Tecnológico (CNPq) (PIBIC). 


\section{Referências Bibliográficas}

ALBUGAMI, S. S. (2016). Developing a strategic approach to ict implementation in saudi secondary schools (Order No. 10589326). Available from ProQuest Dissertations \& Theses Global. (1885877026). Retrieved from https://search.proquest.com/docview/1885877026?accountid=26642

ALMERICH, Gonzalo et al. Las necesidades formativas del profesorado en tic: Perfiles formativos y elementos de complejidad. Relieve, Valência, v. 17, n. 2, p.1-28, jan. 2011. Disponível em: 〈http://www.uv.es/RELIEVE/v17n2/RELIEVEv17n2_1.htm>. Acesso em: 19 set. 2017.

CETIC.BR (2017, 10 October). Ferramenta de Visualização de Dados. Recuperado em 10 de outubro, 2017, do http://cetic.br/

FONSECA, J. J. S. Metodologia da pesquisa científica. Fortaleza: UEC, 2002. Apostila. GIL, A. C. Como elaborar projetos de pesquisa. 4. ed. São Paulo: Atlas, 2007.

HENTON, M. C. (2016). Technology integration practices of faculty in the louisiana community and technical college system (Order No. 10307801). Available from ProQuest Dissertations \& Theses Global. (1871305453). Retrieved from https://search.proquest.com/docview/1871305453?accountid=26642

IBGE. Estatísticas. Disponível em: <http://downloads.ibge.gov.br/downloads_estatisticas.htm>. Acesso em: 08 ago. 2016.

MALTEMPI, Marcus Vinicius; MENDES, Ricardo de Oliveira. Tecnologias digitais na sala de aula: por que não?. In: lisboa, Instituto de Educação da Universidade de (Ed.). Atas do IV Congresso Internacional das TIC na Educação: Tecnologias digitais e a Escola do Futuro. Lisboa: Instituto de Educação da Universidade de Lisboa, 2016. p. 87-97. Disponível em: <http://ticeduca2016.ie.ulisboa.pt/?page_id=1369>. Acesso em: 19 set. 2017.

MINAYO, Maria Cecília de Souza. Pesquisa Social. Teoria, método e criatividade. 18 ed. Petrópolis: Vozes, 2001

NICOLETE, Priscila Cadorin et al. Mobile remote experimentation applied to Basic Education. 2015 3rd Experiment International Conference (exp.at'15), [s.1.], p.266-271, jun. 2015.

OLIVEIRA, Lorena Alves de; FRANCO JUNIOR, Milton Tomaz. A inserção das tecnologias da informação e comunicação nas escolas. In: Simpósio Internacional de Educação a Distância, 1., 2016, São Carlos. SIED:EnPED:2016. São Carlos: Sead, 2016. p. 1 - 11.

PRESBY, B. (2017). Barriers to reducing the digital-use divide as perceived by middle school principals (Order No. 10268273). Available from ProQuest Dissertations \& Theses Global. (1887122371). Retrieved from https://search.proquest.com/docview/1887122371 ?accountid=26642

PRODANOV, C. C.; FREITAS, E. C. Metodologia do trabalho científico: métodos e técnicas da pesquisa e do trabalho acadêmico - 2. ed. - Novo Hamburgo: Feevale, 2013 RESTIVO, Maria Teresa; ALVES, Gustavo R. Acquisition of higher-order experimental skills through remote and virtual laboratories. In: DZIABENKO, Olga; GARCÍA-ZÚBIA, Javier. IT Innovative Practices in Secondary Schools: Remote Experiments. Deusto: Deusto, 2013. Cap.13. p.321-323.

ROCHADEL, Willian et al. Ensino a Distância na Educação Básica: A Integração Pedagógica de Jogos Digitais em Ambientes Virtuais. E-tech: Tecnologias para 
Competitividade Industrial, Florianópolis, v. 9, n. 1, p.32-54, jul. 2016. Disponível em: <http://revista.ctai.senai.br/index.php/edicao01/article/view/824/427>. Acesso em: 07 dez. 2017.

RODRIGUES, Ana Luisa. A integração pedagógica das tecnologias digitais na formação ativa de professores. In: LISBOA, Instituto de Educação da Universidade de (Ed.). Atas do IV Congresso Internacional das TIC na Educação: Tecnologias digitais e a Escola do Futuro. Lisboa: Instituto de Educação da Universidade de Lisboa, 2016. p. 1320-1333. Disponível em: <http://ticeduca2016.ie.ulisboa.pt/?page_id=1369>. Acesso em: 18 set. 2017

SILVA, Ormenzina Garcia da; NAVARRO, Elaine Cristina. A RELAÇÃO PROFESSOR-ALUNO NO PROCESSO ENSINO -APRENDIZAGEM. Revista Eletrônica da Univar, [s.i.], v. 3, n. 8, p.95-100, dez. 2012.

UNESCO, ICT competency standards for teachers: policy framework. Paris: UNESCO, 2008. 